PoS $\quad \begin{aligned} & \text { PROCEEDINGS } \\ & \text { OF SCIENCE }\end{aligned}$

\title{
Foreword to the XXth Hadron Collider Physics Symposium
}

\author{
E. Augé* \\ IN2P3-CNRS, Paris, France \\ E-mail: eauge@admin.in2p3.fr \\ S. Bertolucci ${ }^{\dagger}$ \\ CERN, Geneva, Switzerland \\ E-mail: Sergio.Bertolucci@cern.ch
}

Foreword to the Evian 20th Hadron Collider Physics Symposium

XXth Hadron Collider Physics Symposium

November 16 - 20, 2009

Evian, France

*Symposium Co-Chair.

${ }^{\dagger}$ Symposium Co-Chair. 
The HCP09 conference which took place in Evian between November 16th and November 20th 2009 is the 20th edition of a series which started as a Topical Conference on Hadron Collider Physics, merged in 2005 with the LHC Symposium in order to bring together the Tevatron and LHC communities.

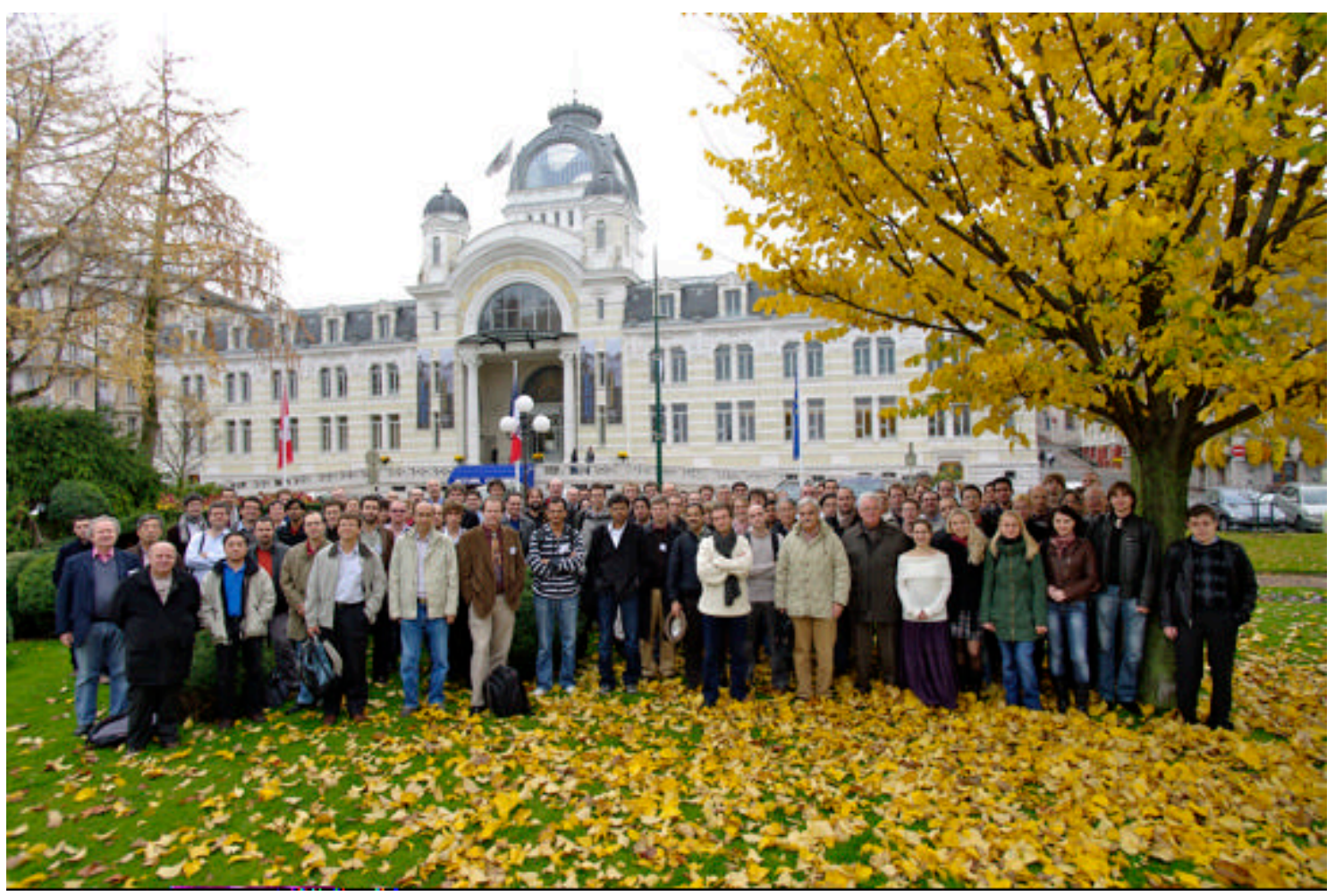

Figure 1: Symposium participants in front of the Palais de Lumière

One clear initial ambition of this conference was to present and discuss the first results from the experiments at the LHC. We were even secretly hoping for signs of physics beyond the standard model. This major event would have remained for posterity as the first milestone of a new era in physics and was worth a grand celebration. Both CERN and the French HEP community were proud to organise it close to CERN. Things did not happen as anticipated and HCP09 is indeed the last conference before LHC: beams were circulating in the machine during the week-end following the conference. However, the program committee of the conference and the speakers turned a difficult challenge into a real success.

The conference has been clearly divided into sessions according to scientific topics (Top quark, New phenomena, Higgs search, Heavy Ions, ...). In each session, LHC talks were mixed with talks from ongoing experiments, and emphasis was put on the present and short term future. This showed to which large extend the LHC experiments represent the next step in our research. Ongoing experiments, in particular CDF and D0, progressing fast, presented an up to date picture of their results, with some emphasis on some hints for phenomena not described by the Standard Model, and on their quest for the Higgs boson whereas the LHC collaborations showed how the detectors are now calibrated and ready to take data, while physicists are ready to analyse them. The last 
afternoon of the conference was entirely devoted to detailed presentations of the future of particle physics in the three big regions of the world, together with a very broad view of the current open questions, and ways to address them in the long term.

The conference was hosted in the Palais de Lumière in Evian, and we wish to thank Monsieur M. Francina, mayor of Evian, for his hospitality and the team of the Tourism Office for the way they made everything become easy for us.

In the name of the institutions co-organizing this conference, we would like to thank all speakers and all those participants to the poster session. We would also like to very warmly thank the program committee and the international advisory committee: the scientific success of the conference results from the pertinent decisions made by those committees. Another ingredient of the success has been the quality of the practical organisation, as well as the way to welcome the participants. We would like to thank the team which took care of the secretariat during the preparation phase, and of the reception desk during the conference. Special thanks should go to P. RoyoleDegieux and G. Boudoul, who took care of the communication and to P. Salati, who gave a public conference in Evian. Special thanks also to O. Drevon, who provided the webcasting and the video recording of the conference. We naturally relied on the local organizing committee for the overall organisation of the conference, but we would like to give special thanks to those members of the committee who took care of the scientific secretariat of each session and of the edition of the proceedings, as well as to those who accepted the difficult task of forming a jury for the poster prize. Out of the 42 high quality posters in competition, the ones from A. Paramonov and from E. Pueschel were selected as the best ones. In the end, our warmest thanks go to the chairmen of the local organising committee, who accepted to devote a large fraction of their time and energy to the overall coordination of the organisation: D. Jacobs, E. Tsesmelis and L. Dobrzynski.

\section{Program Committee :}

D. Charlton - K. Ellis - D. Fournier - P. Jenni - A. Juste - S. Myers - T. Nakada - K. Pitts -

K. Safarik - Y. Sirois - P. Sphicas - J. Stirling - T. Virdee

\section{Scientific secretaries :}

G. Boudoul - A. Cerri - T. Christiansen - C. Goy - T. Koffas - A. Lucotte - L. Malgeri -

D. Rousseau

\section{Poster Prize jury :}
A. Cerri - C. Goy - S. Lammers - L. Malgeri - S. Pranko - D. Rousseau

\section{Scientific secretariat and practical organisation :}

M. Berthier - N. Bleesz-Griggs - C. Demirdjian - P. Mage-Granados - C. Potter -

A. Vignes-Magno 